\title{
Pacific Ocean Neutrino Experiment (P-ONE): prototype line development
}

\section{Christian Spannfellner ${ }^{a, *}$ and Matthias Danninger ${ }^{b}$ on behalf of the P-ONE Collaboration}

(a complete list of authors can be found at the end of the proceedings)

\author{
${ }^{a}$ Technical University Munich, Physics Department, \\ James-Franck-Str. 1, Garching, Germany \\ ${ }^{b}$ Simon Fraser University, Department of Physics, Burnaby, British Columbia, Canada \\ E-mail: christian.spannfellner@tum.de, matthias_danninger@sfu.ca
}

The Pacific Ocean Neutrino Experiment (P-ONE) is a new initiative to construct one of the world's largest neutrino detectors in the deep Pacific Ocean off the coast of British Columbia, Canada. Located in the Cascadia Basin region, P-ONE builds on a number of key strengths within the Canadian oceanographic community. The Cascadia Basin monitoring site is part of the NEPTUNE observatory of Ocean Networks Canada (ONC), which provides power and data connections to various deep ocean sites, accessible to experiments. In cooperation with ONC, the collaboration successfully deployed two pathfinder experiments, the STRAW projects, in 2018 and 2020, respectively. These pathfinder mooring lines aim to measure the optical and ambient background characteristics of the Cascadia Basin in a depth of $2660 \mathrm{~m}$. The P-ONE prototype line is the successor of these mooring lines and the next step towards the P-ONE neutrino observatory. The main objective of the prototype line lies in the construction, deployment, and operation of a complete P-ONE mooring line as a proof of concept of the individual components. This line will comprise of P-ONE digital optical modules to measure the emerging Cherenkov radiation by neutrino-induced processes and P-ONE calibration devices to provide in-situ calibration of the detector. The prototype line will be complemented by external geometry calibration units to verify the envisioned calibration principles.

$37^{\text {th }}$ International Cosmic Ray Conference (ICRC 2021)

July 12 th - 23rd, 2021

Online - Berlin, Germany

\footnotetext{
${ }^{*}$ Presenter
} 


\section{The Pacific Ocean Neutrino Experiment}

The Pacific Ocean Neutrino Experiment (P-ONE) [1] is a proposed cubic kilometer scale neutrino telescope to be deployed off the coast of Vancouver Island, Canada. The project will detect atmospheric and astrophysical neutrinos using the Cherenkov emission from secondary particles produced in neutrino interactions. The main goal of P-ONE is to advance the field of neutrino astronomy, extending the cosmic frontier at the highest energies to reveal previously unknown astronomical phenomena, collect data to test fundamental physics at the PeV scale, and provide crucial information for multi-messenger follow up observations. P-ONE leverages the expertise in deep-sea operations from Ocean Networks Canada (ONC) which is an unique oceanographic observatory that offers an excellent opportunity for the construction of a large volume neutrino telescope. The Cascadia Basin at a depth of 2660 meters has been selected to host P-ONE and is located within ONC's North East Pacific Time-series Underwater Networked Experiment (NEPTUNE) observatory. This observatory spans an $800 \mathrm{~km}$ long loop of fibre optical cable, allowing a data stream of up to $10 \mathrm{~Gb} / \mathrm{s}$ accessible at five nodes with a power distribution of $9 \mathrm{~kW}$ respectively. P-ONE will complement the existing network of neutrino telescopes around the globe (Antares [2], KM3NeT [3], GVD Baikal [4], IceCube [5]) and significantly contribute to a complete neutrino sky coverage at the PeV scale [6].

P-ONE is being designed for a primary purpose of detecting astrophysical neutrinos at multi$\mathrm{TeV}$ energies with excellent directional and energy reconstruction for neutrino events, with a special focus on horizontal tracks. Another key aspect of the design is a modular structure that allows for installation of varying size and easy scalability of the array in different stages. A single P-ONE cluster will consist of 10 strings with 20 optical modules each. The optical properties of the Cascadia Basin are currently being studied by two pathfinder experiments, Strings for Absorption Length in Water (STRAW) and STRAW-b, deployed in 2018 and 2020. These mark already two milestones of the P-ONE project [7], [8]. Preliminary highlights from the STRAW data include a measured attenuation length of $32 \pm 1 \mathrm{~m}$ (at a wavelength of $465 \mathrm{~nm}$ ), in-situ measurement of the ambient backgrounds from radioactive potassium decays, and measurement of low levels of irradiance produced by the bioluminescence at the P-ONE site, ranging from $10^{-11}$ to $10^{-9} \mathrm{~W} / \mathrm{m}^{2}$ [9]. The next milestone towards P-ONE is the development of a prototype mooring line, which will be discussed in these proceedings.

\section{The P-ONE prototype line concept}

P-ONE is currently envisioned as a segmented structure, consisting of 7 clusters with 10 mooring lines each. Each line will include 20 optical modules of two different types: a calibration module and an optical receiver module. The P-ONE prototype line aims to be the blueprint for the P-ONE moorings and follows three objectives: First, the development and construction of the optical modules together with the mechanical mounting structure. Second, the refinement of the deployment strategy and the necessary mechanical deployment support structures. This will allow the collaboration to reach an efficient deployment of several mooring lines during each deployment cruise, guaranteeing a rapid construction of each individual P-ONE cluster. Third, the initial development of around 16-17 optical and 3-4 calibration modules on an instrumented line with 
a planned length of 750 to $1000 \mathrm{~m}$. The optical instruments rely heavily on an unimpaired field of view of the photosensors and the integrating spheres of the calibration module, respectively. Time synchronization between the individual modules, moorings, and clusters of P-ONE will be based on fiberoptical cables. The collaboration is currently exploring options for the most suitable data transfer protocol. One such option is the TRBnet protocol that is based on the Trigger-Readout-Board (TRB), originally developed for collider experiments by GSI, Darmstadt. Preliminary tests show promising results with picosecond-scale resolution. In addition, the predecessor systems are being used in the P-ONE pathfinder experiments, STRAW and STRAW-b. Nonetheless, further tests will be conducted and different technologies evaluated to identify the most suitable system. The prototype line will be accompanied by up to three calibration units. These units are shorter mooring lines consisting of one to two calibration modules each. Such additional lines will enable full scale tests of the time synchronization and the precision calibration system. This system is based on the emission of a high-intensity nanosecond light pulse by the calibration modules, which illuminates the surrounding modules within a cluster and offers the possibility to monitor the detector geometry, eventual changes in time, ageing effects, and changes of the optical properties. These short calibration lines will be recovered once the first P-ONE cluster is fully deployed.

Entanglement and damage of individual components pose a significant threat when deploying $1000 \mathrm{~m}$ mooring lines. The P-ONE collaboration is currently looking into the possibility to combine the mechanical backbone with the hybrid electro-optical cable. This will simplify the structure of the line, reducing the risk of failure and facilitate preparations and handling during deployment.
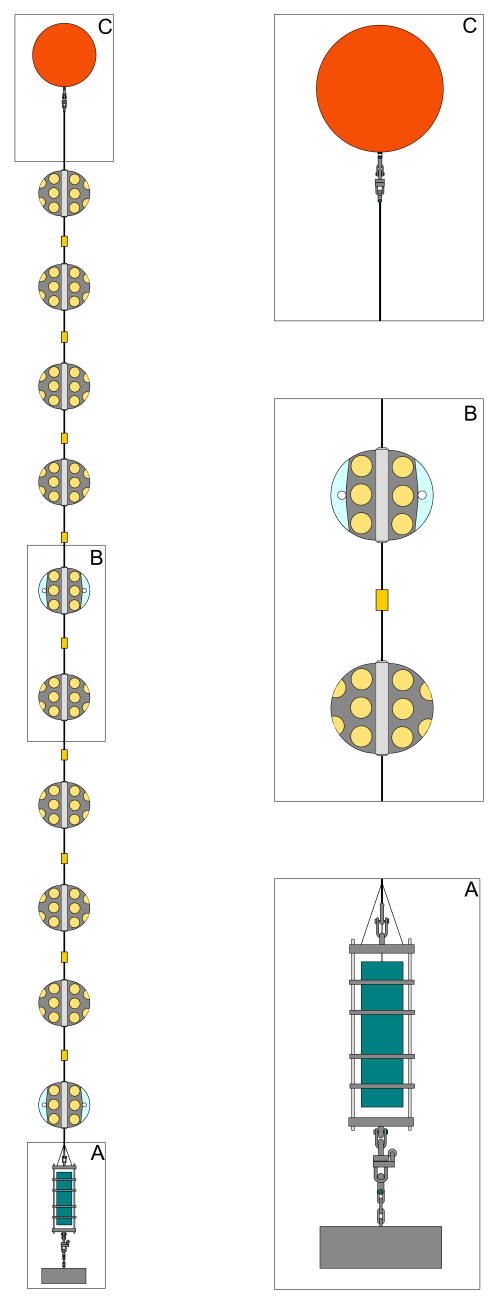

Figure 1: Concept of the P-ONE prototype line, depicting 10 of the envisioned 20 optical modules.

The instrument mountings must serve as robust, safe, and long-lasting connection to the mechanical backbone of the mooring, while still keeping the field of view unimpeded. Possible solutions are currently investigated and range from additional titanium cylinders between the hemispheres to tightly-fitting mooring frames. Regardless of the final approach, the mounting will be designed closely alongside the backbone cable and the instrument core. The concept of the prototype line is shown in Fig. 1, the illustration depicts the potential intermediate titanium flanges between the glass hemispheres.

Fig. 2 displays a cluster, consisting of 10 moorings, and the currently planned P-ONE detector configuration. 

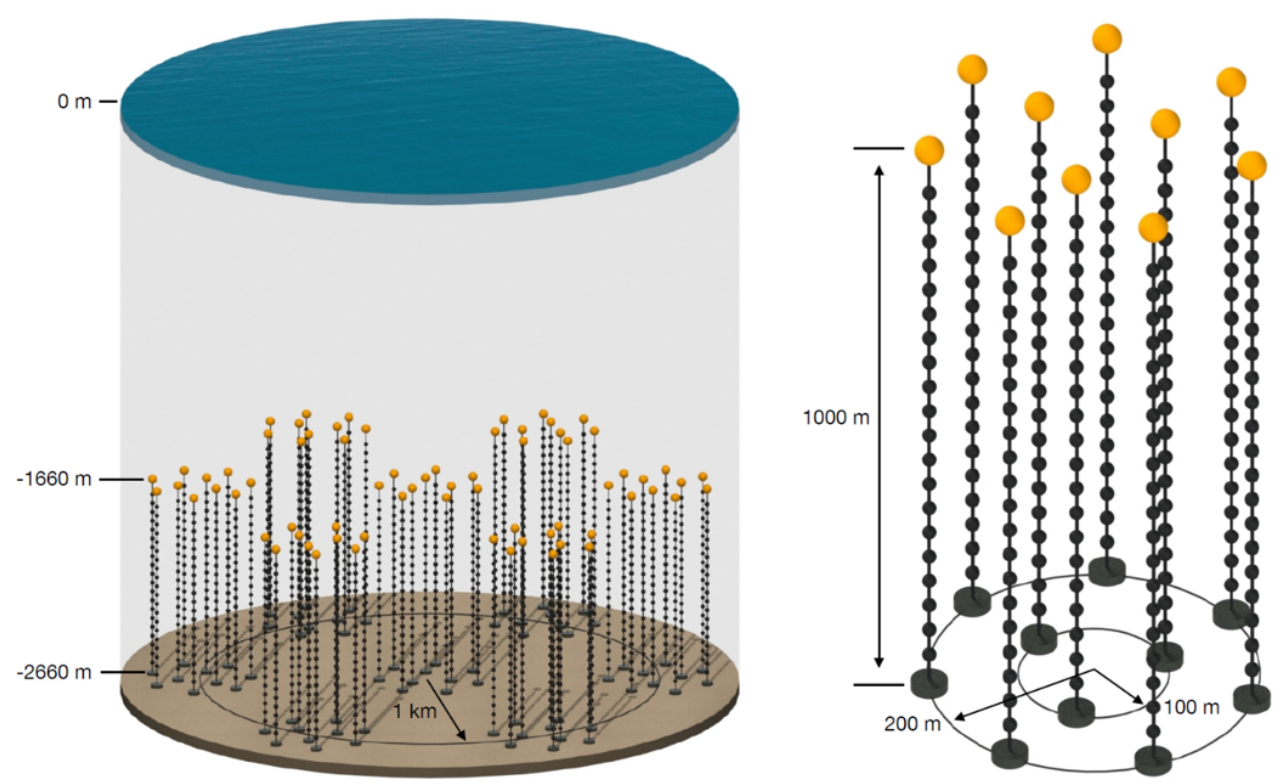

Figure 2: Illustration of the P-ONE configuration. The left side shows the complete detector configuration, consisting of 7 clusters. An individual cluster with 10 mooring lines is depicted on the right. Picture by courtesy of K. Holzapfel.

\section{The P-ONE optical module}

The P-ONE optical module will have a multi photo-multiplier tube (mPMT) configuration in order to achieve the best possible performance within the high light-background environment of the deep Ocean $(\mathrm{O}(10) \mathrm{kHz}$ per PMT). Photons from slow biological processes are not correlated on a nanosecond timescale, which allows the strong suppression of this background by requiring nanosecond-scale coincidences of photon hits in two or more PMTs. This coincidence is expected for neutrino-induced flashes of Cherenkov light, enabling a strong discrimination of signal from background events at the trigger level. The photosensors in the P-ONE optical modules will be PMTs with a diameter between 3-3.5 inches. At the current development stage, candidate PMTs for the optical modules are being evaluated at calibration setups at the University of Alberta and the Technical University Munich. Performing the PMT evaluation at two locations allows independent verification of key PMT parameters of the candidate PMTs. In this context, the prototyping of electronics, reflectors, and the internal mounting structure is in full progress.

\subsection{PMT selection}

The planned set of tests include measurements of dark rate, angular acceptance, transit-time and transit-time-spread, gain, single photo-electron resolution, and quantum efficiency. Both, a laser and a diffuse light source will be used for these tests. The PMT and a calibrated monitoring photodiode are stored in a dark box and mounted on an optical table. The utilization of two rotation stages allows the scanning of the photocathode area in azimuth and zenith for the angular measurements. The readout is performed via an oscilloscope and a picoamperemeter. In addition 
to the initial PMT selection, both setups will be further used to calibrate the individual PMTs before integration in the optical modules. To ensure an easy and fast calibration, the necessary readout and supplementary electronics are remote-controllable, which enables a scan of the PMTs on a plug-and-play basis via distinct measurement scripts.

\subsection{Transparent reflectors and internal mounting}

One of the most vital criteria of the optical module is its effective photosensor area. A full surface coverage of the glass spheres with PMTs is impossible due to space constraints inside the module. The additional implementation of reflective cones around the PMTs is the most efficient way to increase the effective instrumented area. In addition, the PMTs have to be optically coupled to the glass housing to minimize refraction effects, which would impair the photon detection and result in a more challenging event reconstruction. In an attempt to address both challenges, we are studying the use of pre-moulded gel pads. These pads are optically coupled to the individual PMTs and act as reflective cones once the incident photons are in the total internal reflection (TIR) regime. Photons, which do not hit the PMT, traverse the gel pad and can be detected by a neighbouring PMT by entering the respective subsequent gel pad. Non-transparent reflectors potentially reflect photons with an unfavoured incident angle out of the module. Transparent reflectors, however, offer a weaker collection efficiency for photons from a perpendicular direction. While the overall efficiency of both types of pads for one PMT is comparable averaged over all incident angles, the transparent reflector performs better in a multi-PMT module (see Fig. 4). The casting of the gel pads has already been tested, yielding promising results in an aluminum mould for different optical gel variations. The internal mounting of the PMTs and supplementary electronics is based on a modular approach. The PMTs will be mounted in a spring-loaded configuration to cope with

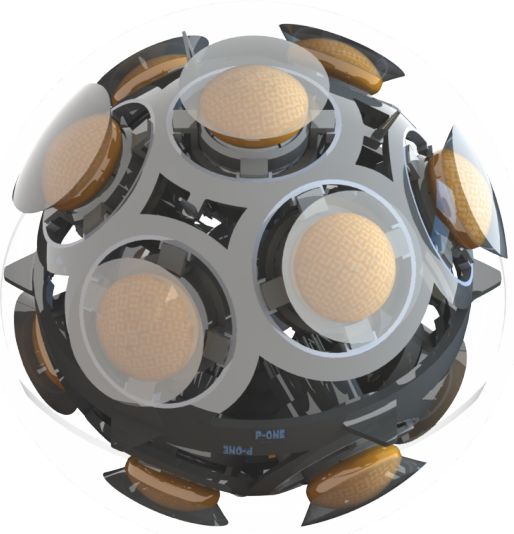

Figure 3: Peliminary internal mounting concept of the P-ONE optical module. The illustration depicts the transparent gel pads and the mounting structure. The PMTs will be mounted by a click-in mechanism to the internal mounting frame.

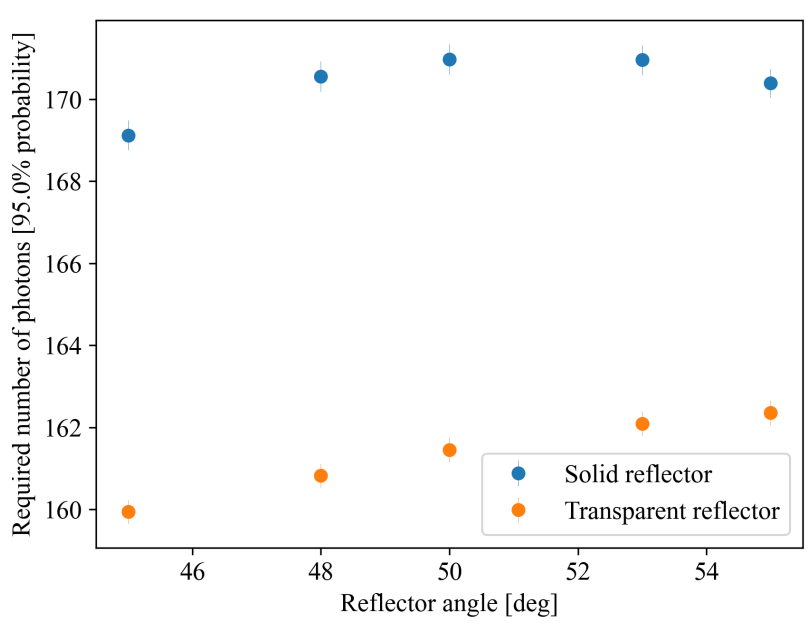

Figure 4: Average required minimum number of photons for an arbitrary direction, which are needed for the optical module to have at least three PMTs detecting one or more photons with a probability of 95\%. Averaged over all reflector angles, the transparent reflector performs $5.7 \%$ better than the solid reflector. 
the emerging temperature gradients and to optimize the optical coupling to the glass housing. A preliminary concept is shown in Fig. 3. The electronic boards for readout and time synchronisation can either be mounted in the instrument core or incorporated in an intermediate flange between both glass hemispheres. Note, this intermediate flange is not displayed in the concept illustrations.

\section{The P-ONE Calibration module}

The optical properties and the geometry of the P-ONE sensor array need to be constantly monitored during operation in order to obtain the best possible data and angular resolution. The specialized calibration modules that complement the standard P-ONE modules within the array will serve this purpose. The calibration modules emit nanosecond isotropic flashes of light, which are detected by the surrounding optical modules, facilitating the measurement of light attenuation via the flash intensity. This concept has been proven with the STRAW experiment [7]. As a key novelty for neutrino telescopes worldwide, the PONE collaboration will develop a system that allows one to base the entire geometry calibration on this module, exploiting the excellent timing properties of the system. All other water-based neutrino experiments utilize a separate acoustic calibration system. Such an additional system, however, adds complexity and costs to the experiment and has the effect of potentially interfering with other marinebiology studies ongoing at the acoustic-quiet zone of the Cascadia Basin site. The light output of the flashers will be monitored in-situ by built-in optical sensors. This gen-

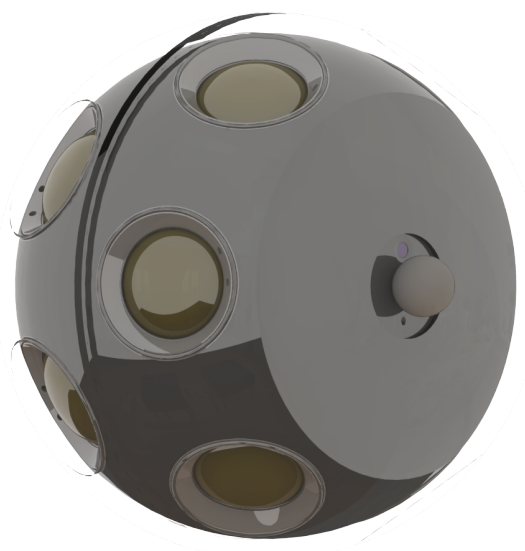

Figure 5: Illustration of the preliminary concept of the P-ONE calibration module. Please refer to the text for more information. eral concept and the required electronics have already been explored in great detail in the design of the POCAM for the IceCube Upgrade and STRAW [7], [10], [11]. The proven POCAM design will form the basis for successful adaptation and further development of the P-ONE calibration module.

\subsection{Electronics}

The key aspect of the calibration module is the emission of nano-second light pulses. For this, two different pulse drivers are used. The Kapustinsky circuit relies on the controlled discharge of a capacitor initiated by a trigger input pulse. The intensity and pulse width can be altered by the values of the capacitor and an inductor, which effectively counteracts the current flow through the emitting diode [12]. The circuit is well established and reliable. However, the low current does not allow the utilization of laser diodes. To accomodate higher currents, a different flasher circuit based on a LMG1020 gate driver is used. This driver switches a Gallium-Nitride field effect transistor (GaN-FET) and consequently initiates the discharge of a capacitor bank [13]. This circuit allows the variation of the pulse width and intensity by varying the input trigger pulse width or the capacitor bank and is capable of reaching very high intensities. The achieved dynamic range lies between $10^{7}$ to $10^{11}$ photons, depending on the selected emitter and pulse width [10]. By controlling the input pulse width via an FPGA-based circuit, the LMG1020 driver circuit offers a 
very versatile platform, capable of reaching both extremely high and low intensities. This enables the calibration of modules at larger distances and avoids saturation of close-by modules. To reach an isotropic emission profile, the emitter matrix that consists of the LEDs and laser diodes, is coupled to an integrating sphere made of optical teflon [10]. This allows in addition the in-situ monitoring within the calibration module, which is performed by a photodiode, with a specifically designed transimpedance amplifier readout, and a SiPM for lower intensities [10]. The SiPM readout further allows the measurement of the pulse on-set time as part of its pulse response is used to switch an ultra-fast transistor and record the rising edge within a TDC. The remainder of the pulse is fed to a secondary amplifier for a conventional charge measurement.

\subsection{Enclosure concept}

The calibration electronics and supplementary mechanical parts will be enclosed in the instrument housing of the optical modules, to allow the seamless integration on the mooring line. This will simplify the design for different mounting strategies, reduce deployment complexity and technical challenges. The integration of additional photosensors into the calibration module is currently considered. This will allow the detection of neutrino-induced events at the position of the calibration modules, effectively avoiding blind spots in the detector volume. To ensure the isotropic emission of the light pulses with an altered housing, the emission profile will be simulated and adapted shapes of the isotropizing PTFE spheres will be studied in a dark chamber. The possibility of temporarily switching off the high voltage (HV) of the PMTs in the calibration module to prevent damage has yet to be studied. It is also possible that power cycling the HV will lead to a temporarily increased dark noise rate of the PMT. Finally, long term aging effects on the PMTs are also possible. Nevertheless, all those effects need to be, and will be, studied thoroughly. The P-ONE collaboration is confident that these challenges can be addressed. A possible setup of the module is shown in Fig. 5.

\section{Conclusions}

The current generation of neutrino telescopes work based on the same principles: instrument huge volumes of reasonably dense and transparent media with fast, large area light sensors to detect flashes of Cherenkov light from cascades of secondary particles. The reconstruction of the neutrino events in terms of background rejection, energy, and directional reconstruction heavily relies on the knowledge of the optical properties of the medium. Equally crucial for a strong detector performance is the precise calibration of its geometry and the sensitivity of individual light sensors. To launch P-ONE, the next milestone is to deploy the very first instrumented line in order to verify the working principle of the newly developed optical instruments, the deployment technique, and to serve as the blueprint of the neutrino telescope's mooring lines. The development of this first line, which will involve 20 optical instruments distributed along an approximately $1000 \mathrm{~m}$ long mooring, started early 2021 and is in full progress. 


\section{References}

[1] M. Agostini, M. Böhmer, J. Bosma, K. Clark, M. Danninger, C. Fruck et al., The pacific ocean neutrino experiment, Nature Astronomy 4 (2020) 913.

[2] C. Perrina, "The antares neutrino telescope."

[3] A. Margiotta, The km3net deep-sea neutrino telescope, Nuclear Instruments and Methods in Physics Research Section A: Accelerators, Spectrometers, Detectors and Associated Equipment 766 (2014) 83.

[4] A.D. Avrorin, A.V. Avrorin, V.M. Aynutdinov, R. Bannash, I.A. Belolaptikov, V.B. Brudanin et al., Baikal-gvd: first results and prospects, EPJ Web of Conferences 209 (2019) 01015.

[5] M.G. Aartsen, M. Ackermann, J. Adams, J.A. Aguilar, M. Ahlers, M. Ahrens et al., The icecube neutrino observatory: instrumentation and online systems, Journal of Instrumentation 12 (2017) P03012.

[6] L.J. Schumacher, M. Huber, M. Agostini, M. Bustamante, F. Oikonomou and E. Resconi, PLEvM: A global and distributed monitoring system of high-energy astrophysical neutrinos, PoS ICRC2021 (2021) 1185.

[7] M. Boehmer, J. Bosma, D. Brussow, L. Farmer, C. Fruck, R. Gernhäuser et al., Straw (strings for absorption length in water): pathfinder for a neutrino telescope in the deep pacific ocean, Journal of Instrumentation 14 (2019) P02013.

[8] I.C. Rea, K. Holzapfel and A. Baron, P-ONE second pathfinder mission: STRAW-b, PoS ICRC2021 (2021) 1092.

[9] N. Bailly, J. Bedard, M. Bohmer, J. Bosma, D. Brussow, J. Cheng et al., Optical analysis of the Pacific Ocean Neutrino Experiment (P-ONE) site using data from the first pathfinder mooring, PoS ICRC2021 (2021) 1160.

[10] F. Henningsen, M. Böhmer, A. Gärtner, L. Geilen, R. Gernhäuser, H. Heggen et al., A self-monitoring precision calibration light source for large-volume neutrino telescopes, Journal of Instrumentation 15 (2020) P07031.

[11] N. Khera and F. Henningsen, POCAM in the IceCube Upgrade, PoS ICRC2021 (2021) 1049.

[12] J.S. Kapustinsky, R.M. DeVries, N.J. DiGiacomo, W.E. Sondheim, J.W. Sunier and $\mathrm{H}$. Coombes, A fast timing light pulser for scintillation detectors, Nuclear Instruments and Methods in Physics Research Section A: Accelerators, Spectrometers, Detectors and Associated Equipment 241 (1985) 612.

[13] EPC, Development board epc9126/epc9126hc quick start guide, 2019. 


\section{Full Authors List: P-ONE Collaboration}

Nicolai Bailly ${ }^{1}$, Jeannette Bedard ${ }^{1}$, Michael Böhmer ${ }^{2}$, Jeff Bosma ${ }^{1}$, Dirk Brussow ${ }^{1}$, Jonathan Cheng ${ }^{1}$, Ken Clark ${ }^{3}$, Beckey Croteau ${ }^{1}$, Matthias Danninger ${ }^{4}$, Fabio De Leo ${ }^{1}$, Nathan Deis ${ }^{1}$, Matthew Ens $^{4}$, Rowan Fox ${ }^{1}$, Christian Fruck ${ }^{2}$, Andreas Gärtner ${ }^{5}$, Roman Gernhäuser $^{2}$, Darren Grant ${ }^{6}$, Helen He ${ }^{1}$, Felix Henningsen ${ }^{7}$, Kilian Holzapfel ${ }^{2}$, Ryan Hotte ${ }^{1}$, Reyna Jenkyns ${ }^{1}$, Hamish Johnson ${ }^{4}$, Akanksha Katil ${ }^{5}$, Claudio Kopper ${ }^{6}$, Carsten B. Krauss ${ }^{5}$, Ian Kulin ${ }^{1}$, Klaus Leismüller ${ }^{2}$, Sally Leys ${ }^{8}$, Tony Lin ${ }^{1}$, Paul Macoun ${ }^{1}$, Thomas McElroy $^{5}$, Stephan Meighen-Berger ${ }^{2}$, Jan Michel ${ }^{9}$, Roger Moore ${ }^{5}$, Mike Morley ${ }^{1}$, Laszlo Papp ${ }^{2}$, Benoit Pirenne ${ }^{1}$, Tom Qiu ${ }^{1}$, Mark Rankin ${ }^{1}$, Immacolata Carmen Rea ${ }^{2}$, Elisa Resconi ${ }^{2}$, Adrian Round ${ }^{1}$, Albert Ruskey ${ }^{1}$, Ryan Rutley ${ }^{1}$, Christian Spannfellner ${ }^{2}$, Jakub Stacho $^{4}$, Ross Timmerman ${ }^{1}$, Meghan Tomlin ${ }^{1}$, Matt Tradewell ${ }^{1}$, Michael Traxler ${ }^{10}$, Matt Uganecz ${ }^{1}$, Seann Wagner ${ }^{1}$, Juan Pablo Yañez ${ }^{5}$, Yinsong Zheng ${ }^{1}$

${ }^{1}$ Ocean Networks Canada, University of Victoria, Victoria, British Columbia, Canada.

${ }^{2}$ Department of Physics, Technical University of Munich, Garching, Germany.

${ }^{3}$ Department of Physics, Engineering Physics and Astronomy, Queen's University, Kingston, Ontario, Canada.

${ }^{4}$ Department of Physics, Simon Fraser University, Burnaby, British Columbia, Canada.

${ }^{5}$ Department of Physics, University of Alberta, Edmonton, Alberta, Canada.

${ }^{6}$ Department of Physics and Astronomy, Michigan State University, East Lansing, MI, USA.

${ }^{7}$ Max-Planck-Institut für Physik, Munich, Germany.

${ }^{8}$ Department of Biological Sciences, University of Alberta, Edmonton, Alberta, Canada.

${ }^{9}$ Institut für Kernphysik, Goethe Universität, Frankfurt, Germany.

${ }^{10}$ Gesellschaft für Schwerionenforschung, Darmstadt, Germany. 